\title{
Swept Source Optical Coherence Tomography
}

\section{Compared to Ultrasound and Biomicroscopy for Diagnosis of Posterior Vitreous Detachment}

This article was published in the following Dove Press journal:

Clinical Ophthalmology

\section{Marlene D Wang (D) \\ Cecile Truong \\ Zaid Mammo \\ Syed Amal Hussnain \\ Royce WS Chen}

Department of Ophthalmology, Edward S. Harkness Eye Institute, Columbia University Irving Medical Center, New York, NY, 10032, USA
Correspondence: Royce WS Chen Edward S. Harkness Eye Institute, Columbia University Irving Medical Center, 635 W 165th St, New York, NY, 10032, USA

$\mathrm{Tel}+\mid$ 2I2-342-4570

Email Rc263I@cumc.columbia.edu
Background: Biomicroscopy, B-scan ultrasound imaging, and SD-OCT are all modalities used to characterize a posterior vitreous detachment (PVD). Our objective is to assess the precision of the diagnosis of PVD by SS-OCT.

Methods: This prospective observational study examines ninety-five eyes of forty-nine patients with biomicroscopy, B-scan ultrasound, and SS-OCT for the presence or absence of a complete PVD. All SS-OCT images were reviewed by two retina specialists (RWSC, ZM). All three diagnostic methods were evaluated for agreement by Cohen's kappa statistic. Results: The inter-rater reliability between retina specialists reading the SS-OCT images was $97.9 \%(\kappa=0.957)$. Agreement on PVD status between SS-OCT and biomicroscopy was $85.3 \%(\kappa=0.711)$. Agreement between SS-OCT and B-scan ultrasound was $83.2 \%(\kappa=$ 0.667). Agreement between B-scan ultrasound and biomicroscopy was $87.4 \%(\kappa=0.743)$.

Conclusion: For the diagnosis of complete PVD, SS-OCT allows for high accuracy and agreement between graders.

Keywords: optical coherence tomography, swept source, posterior vitreous detachment

\section{Introduction}

The advent of swept-source optical coherence tomography (SS-OCT) has allowed for more detailed imaging of the vitreous and vitreoretinal interface through improved depth of imaging and scan speed. ${ }^{1-3}$ The relationship between the vitreous and the retina can have implications for the treatment of associated retinal pathology including vitreomacular traction syndrome, macular holes, epiretinal membranes, high myopia, and retinal tears. ${ }^{4}$ In particular, a partial posterior vitreous detachment (PVD) may be related to progression of vitreoretinal disease, whereas a complete PVD may serve as a protective element. ${ }^{5-7}$ Biomicroscopy and B-scan ultrasound imaging are limited by lower resolution, the experience of the operator, and patient cooperation. Time domain and spectral-domain optical coherence tomography (SD-OCT) have proven invaluable in the evaluation of the vitreoretinal interface, ${ }^{8,9}$ but can be limited due to challenges with resolution, depth, and window size. ${ }^{10}$ A recent study examining SD-OCT macular images before and after vitrectomy concluded that this modality has a high negative predictive value (detects attached vitreous accurately), but has a low positive predictive value (detects a complete PVD less accurately). ${ }^{11}$ However, there is also recent evidence suggesting that SD-OCT images including the peri-papillary region have higher levels of inter-rater agreement than those of ultrasonography. ${ }^{12}$ 
SS-OCT has improved depth imaging primarily by using a longer wavelength of $1050 \mathrm{~nm}$, and faster scan speeds, with commercially available instruments starting at an axial scan rate of 100,000 A-scans/second. ${ }^{1}$ These improvements allow for improved depth resolution and the simultaneous detailed imaging of the macula, optic nerve head, choroid, and vitreous structures. ${ }^{13,14}$ SS-OCT has been used to characterize the vitreous structures in healthy adults and children, high myopes, and patients after cataract surgery. ${ }^{4,14-16}$ Widefield OCT imaging has been used to characterize the PVD status throughout various decades of life, suggesting that PVD usually starts in the periphery and progresses towards the fovea. ${ }^{17}$ However, studies evaluating PVD detection with SS-OCT compared to B-scan ultrasound imaging and biomicroscopy are limited. We propose that SS-OCT has high inter-rater reliability, is non-inferior to B-scan ultrasound imaging and biomicroscopy for the diagnosis of PVD, and may be particularly useful for characterizing and detecting a partial PVD due to the enhanced detail of the vitreous-retina interface.

\section{Patients and Methods}

This is a prospective observational study conducted according to the tenets of the Declaration of Helsinki, and approval was obtained by the Institutional Review Board (IRB) of Columbia University (Protocol \# AAAR7199 M00Y01). All patients gave informed consent to be imaged and to have deidentified data used for publication. Patients were enrolled from March 2018 to August 2018.

Consecutive patients at Columbia Doctors' retina practice were included in the study. Patients with vitreoretinal pathologies such as macular degeneration and diabetic retinopathy were included in the study. Patients with a media opacity (such as a dense cataract or vitreous hemorrhage), vitritis, or a history of a previous vitrectomy were excluded from the study.

All eyes that were included in the study underwent an examination first by biomicroscopy at the slit lamp using a Volk 90 D lens, and then subsequently by $10-\mathrm{MHz}$ B-scan ultrasonography ( $\mathrm{I}^{3}$ Innovative Imaging Inc., Business Park Drive Sacramento, CA, USA) by one retinal specialist (RWSC). A determination of PVD status was made at the conclusion of the biomicroscopic exam. A kinetic ultrasound examination by B-scan was then performed in the axial plane under high gain $(90 \mathrm{~dB})$ to maximize visualization of the cortical vitreous. A separate determination of PVD status was made at the conclusion of the kinetic ultrasound. A PVD was considered complete by biomicroscopy if a Weiss ring was visualized. The PVD was considered complete by B-scan if the posterior hyaloid was detached at the optic nerve head. Each patient separately had an SS-OCT 16-mm horizontal line scan including the optic nerve head and macula which focused on the vitreoretinal interface (Plex Elite 9000, Carl Zeiss Meditec Inc. Hacienda Drive, Dublin, CA, USA) and was performed by a trained photographer. The brightness of the SSOCT images was enhanced to optimize visualization of the cortical vitreous. All SS-OCT images were subsequently evaluated for the presence or absence of a complete PVD by two retina specialists (RWSC, ZM) in a masked fashion. Eyes were considered to have a complete PVD by SS-OCT imaging if the posterior hyaloid was detached at the fovea and the optic nerve head. Our sample size achieves $80 \%$ power to detect a true Kappa value $(p<0.05)$. Agreement was measured between the two independent retina specialists (RWSC, ZM) grading the SS-OCT images for the presence or absence of a complete PVD. Agreement among the three modalities was also measured using Cohen's kappa statistic. All statistical analysis was carried out with SPSS statistical software version 25.0 (SPSS Inc, Chicago, Illinois, USA).

\section{Results}

Ninety-five eyes of forty-nine subjects (18 men and 31 women) were included in the study (mean 63.7, range 18-92 years). SS-OCT detected a complete PVD at a significantly greater rate than B-scan $(\mathrm{p}=0.012)$ or biomicroscopy $(p=0.0001)$ (Table 1$)$. There was no statistically significant difference between the rate of PVD detection between B-scan and biomicroscopy ( $\mathrm{p}=$ 0.250 ). In $44.2 \%$ of SS-OCT images, the vitreous was clearly adherent to the retina, and a complete PVD was not identified (Figure 1A and B).

The inter-rater reliability between retina specialists reading the SS-OCT images was $97.9 \%(\kappa=0.957)$. The level of agreement was not significantly different when comparing each modality to a second modality $(\mathrm{p}=$ 0.250). A high level of agreement on PVD status between SS-OCT and biomicroscopy was achieved $(85.3 \%, \kappa=$

Table I Rate of PVD Diagnosis. SS-OCT Diagnosed a Complete PVD at a Significantly Higher Rate Than Ultrasound or Biomicroscopy

\begin{tabular}{|l|l|l|}
\hline Ultrasound & Biomicroscopy & SS-OCT \\
\hline $45.30 \%$ & $41.10 \%$ & $55.80 \%$ \\
$43 / 95$ eyes & $39 / 95$ eyes & $53 / 95$ eyes \\
\hline
\end{tabular}




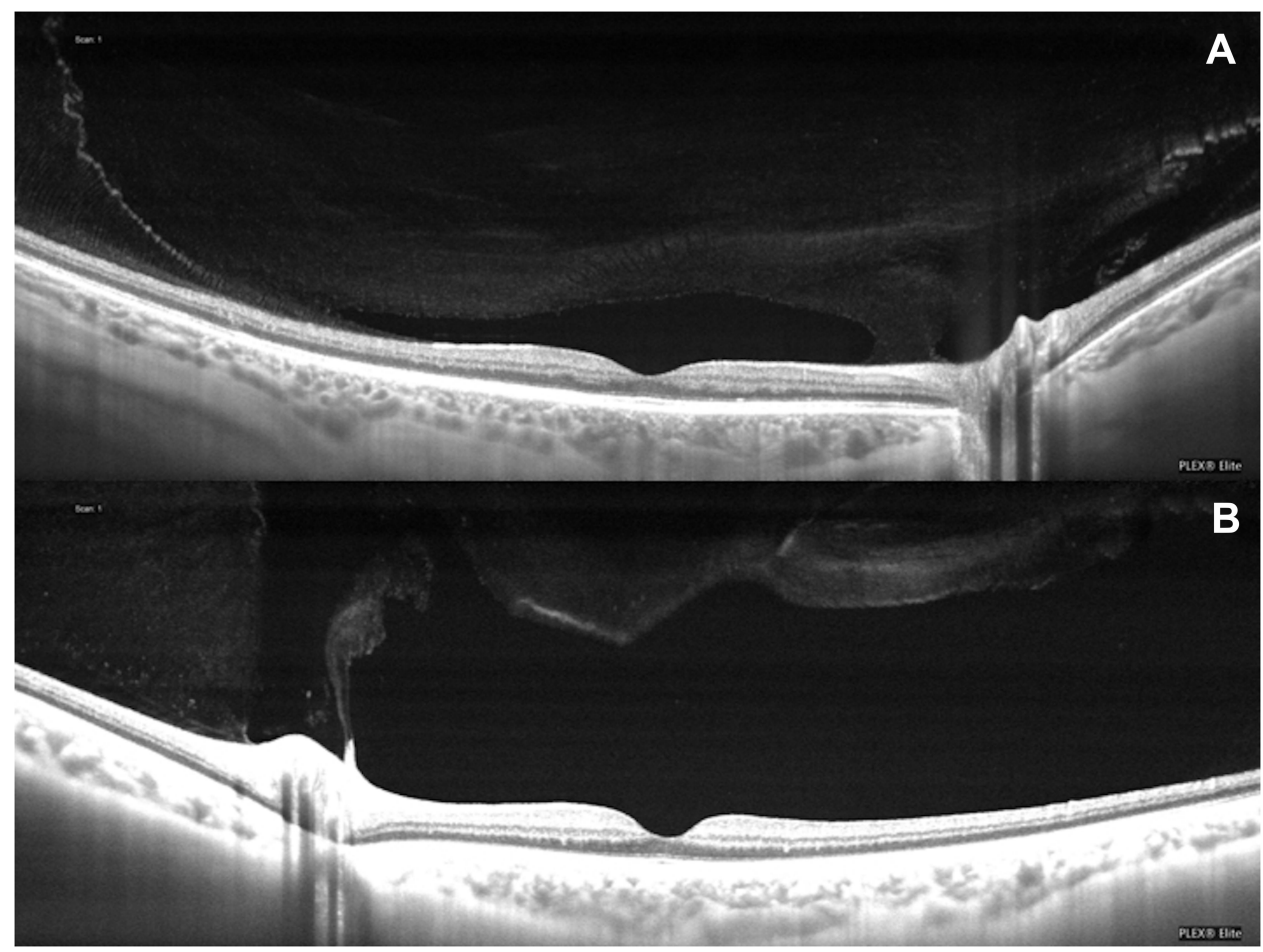

Figure I (A and B) No complete posterior vitreous detachment (PVD) identified by Swept Source Optical Coherence Tomography (SS-OCT). (A) The premacular bursa is visible, and the vitreous remains attached temporally and at the optic nerve head. (B) The vitreous is detached temporally and at the fovea but remains adherent to the optic nerve head.

0.711), and agreement between SS-OCT and B-scan ultrasound was similarly high $(83.2 \%, \kappa=0.667)$. Agreement between B-scan ultrasound and biomicroscopy was found to be comparable at $87.4 \%(\kappa=0.743)$.

In the 16 cases where there were disagreements between SS-OCT and B-scan, the SS-OCT images provided more detailed information that helped to determine the presence $(13 / 16)$ or absence $(3 / 16)$ of a complete PVD (Table 2). Figure 2 shows an instance of complete separation between the vitreous at the optic nerve head and the fovea, although no PVD was detected by B-scan or biomicroscopy. In three cases, a complete PVD was diagnosed by B-scan although SS-OCT identified persistent vitreoretinal adhesions. SS-OCT images in these three cases revealed the presence of hyperreflective opacities in the vitreous body compared to the less reflective cortical vitreous. This anatomical difference appeared on ultrasound as a freely mobile hyper-reflective posterior vitreous interface that simulated a complete PVD (Figure 3A and $\mathrm{B}$ ). When evaluating eyes classified as no PVD by both B-scan ultrasound and SS-OCT, these vitreous body opacities were present in 30/39 eyes. When biomicroscopy was compared to SS-OCT, all disagreements (14/14) were due to PVD being undetected on slit-lamp exam, but present on SS-OCT (Figure 4). There was no association between the disagreement among the three diagnostic modalities and the lens status of the eye $(p=0.987)$.

\section{Discussion}

OCT is a powerful diagnostic tool for evaluating the vitreoretinal interface. Past studies have suggested concerns of a high rate of false-positive PVD diagnoses due to a small window size. ${ }^{5,18}$ A study by Bertelmann et al suggested that a PVD could be falsely diagnosed by SS-OCT when using a $6 \mathrm{~mm}$ window (which does not include the optic nerve head), but refuted by a $12 \mathrm{~mm}$ scan because of persistent vitreous adhesion to the optic nerve head. ${ }^{5,19}$ In some cases, a hyporeflective premacular bursa can be mistaken for a PVD. ${ }^{20}$ Inclusion of the areas peripheral to the fovea, including the optic nerve head, is critical to determining the true PVD status. ${ }^{10}$ A study by Uchino et al examining 209 eyes classifies five stages of a PVD: stage 1, no PVD; stage 2, perifoveal detachment in the superior quadrant; stage 3, perifoveal detachment superior and inferior to the fovea with persistent attachment at the fovea and optic nerve head; stage 4, with detachment from the fovea, but persistent attachment at the optic nerve head; and stage 5, with complete release of the vitreopapillary adhesion. ${ }^{8}$ A recent study by Hwang et al 2020 examines macular 
Table 2 Cases with Discrepancies Between SS-OCT and Ultrasound

\begin{tabular}{|l|l|l|l|l|l|}
\hline & Ultrasound PVD & Biomicroscopy PVD & SS-OCT PVD & Lens Status & Refractive Error \\
\hline I & No & No & Yes & Pseudophakic & +3.5 \\
2 & No & No & Yes & Phakic & -1.5 \\
3 & Yes & No & No & Phakic & +1 \\
4 & Yes & No & No & Pseudophakic & 0 \\
5 & Yes & Yes & Yes & 0 \\
6 & No & Yes & Yes & Phakic & -6.25 \\
7 & No & Yes & Yes & -6.25 \\
8 & No & No & Yes & Phakic & -10 \\
9 & No & No & Yes & Phakic & +2.25 \\
10 & No & No & Yes & Phakic \\
11 & No & No & Yes & Phakic & +3 \\
12 & No & No & Yes & Phakic & -1.25 \\
13 & No & Yes & Yes & Pseudophakic & +1.25 \\
14 & No & No & Yes & Pseudophakic & +1.75 \\
15 & No & No & Phakic & -1.25 \\
16 & No & Phakic & -3.25 \\
\hline
\end{tabular}

SD-OCT images before and after vitrectomy, concluding that this modality has a high negative predictive value (detects attached vitreous accurately), but has a low positive predictive value (detects a complete PVD less accurately). ${ }^{11}$ Our SS-OCT images included a $16 \mathrm{~mm}$ window which extended past both the perifoveal region and the optic nerve head. The size and depth of these images, in combination with a brightened vitreoretinal interface, allowed the raters to confidently identify a complete PVD (no persistent adhesion of the vitreous to either the fovea or the optic nerve head) with a high level of agreement.

In the present study, the level of agreement regarding PVD status was similar for biomicroscopy, B-scan ultrasound, and SS-OCT. In the majority of cases of discrepancies, a complete PVD was visualized on SS-OCT, but no complete PVD was detected by the other methods. On
B-scan, this situation occurred when, despite high gain, the posterior hyaloid could not be visualized floating freely over the retina and optic nerves. On biomicroscopy, the PVD could not be detected perhaps because the vitreous was more liquified, and no clear glial cells could be seen to delineate a Weiss ring. In a few cases, ultrasound classified a PVD as present, but persistent vitreoretinal adhesions were seen on SS-OCT, revealing additional information about the vitreoretinal interface that could not otherwise be detected.

Our study had several limitations that may be addressed in future trials. Due to time constraints, the clinical examination and the ultrasound examination were performed by the same examiner. Ideally, these exams would be carried out by separate examiners. In order to introduce as much standardization as possible to the evaluation process, all biomicroscopic exams were performed

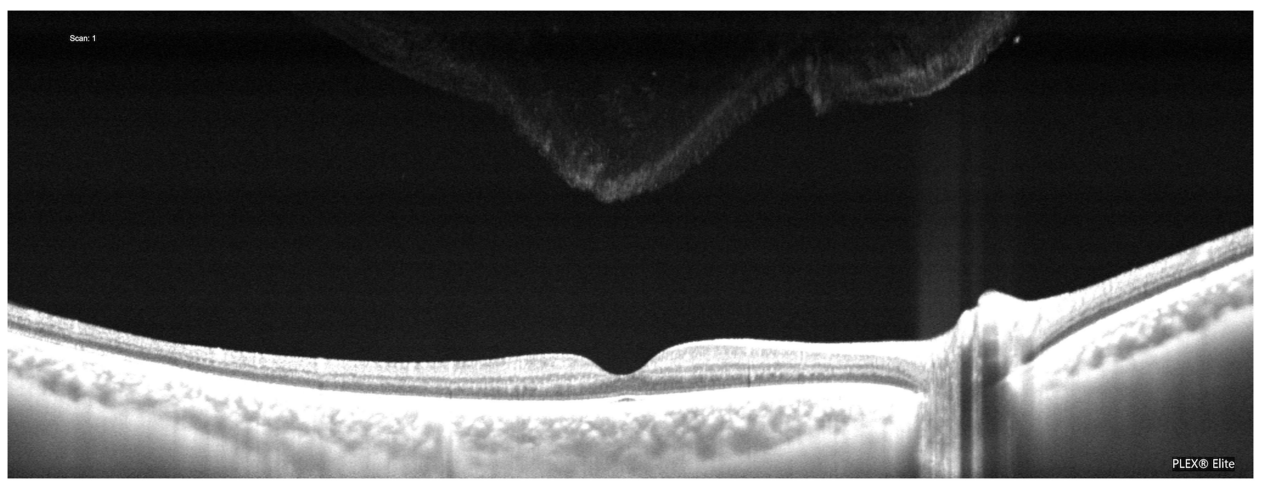

Figure 2 No PVD was identified by ultrasound, however the SS-OCT revealed a complete detachment of the vitreous from the optic nerve head and the fovea. 


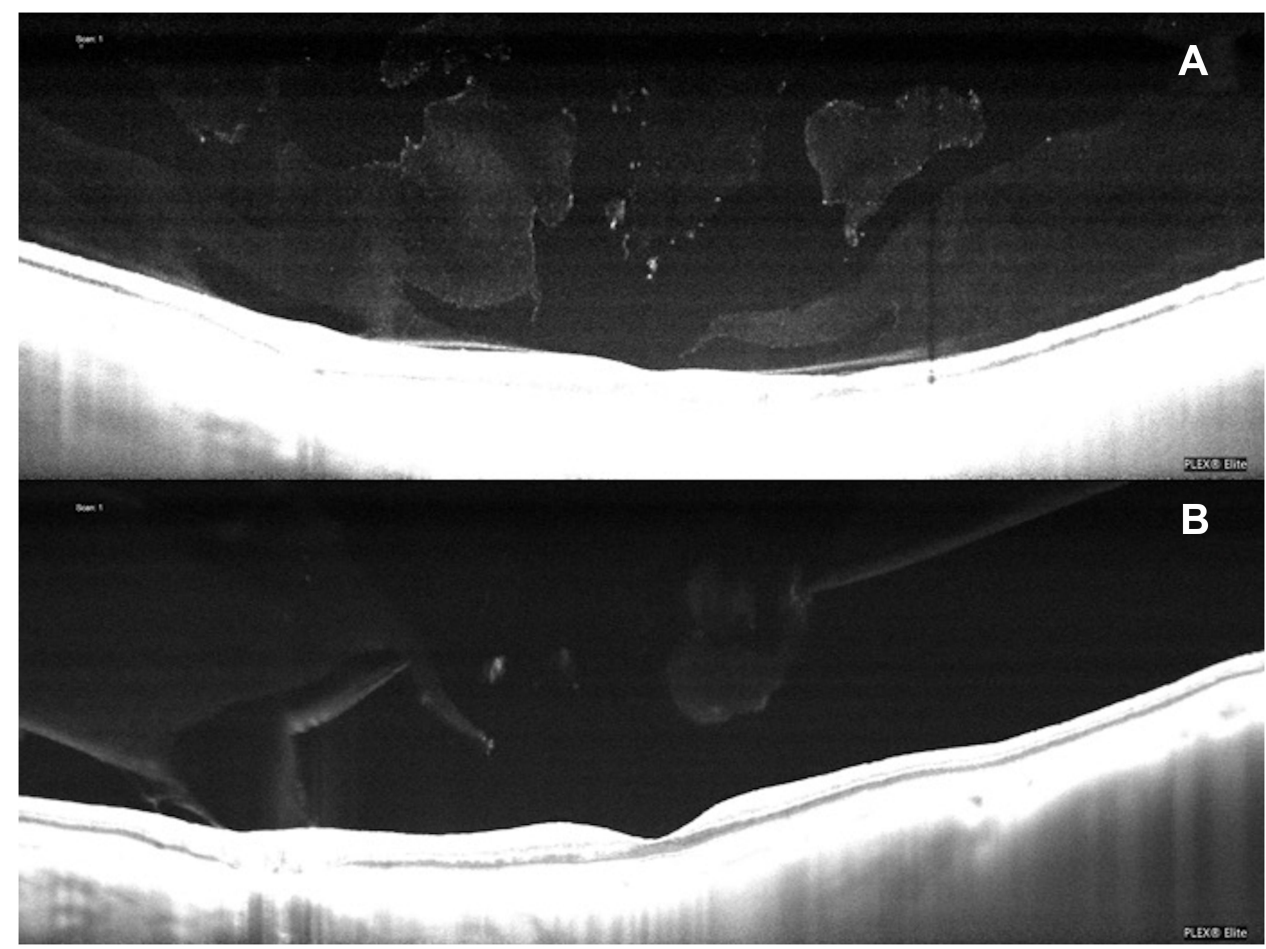

Figure 3 (A) A PVD was identified by ultrasound, however the SS-OCT revealed adhesions of the vitreous at the fovea and the optic nerve head. The optically empty pockets seen within the vitreous of the SS-OCT image may represent a situation that is not easily identified with ultrasound. (B) SS-OCT revealed a small residual adhesion of the vitreous to the optic nerve head that was not identified by ultrasound.

and graded prior to ultrasound examination and grading, and SS-OCT was performed separately by the ophthalmic imaging department. Future studies may also look to compare the interrater reliability of ultrasound, clinical examination, and SD-OCT imaging to that of SS-OCT imaging. We chose our binary grading scheme (complete PVD versus no PVD) as an initial study, but future studies regarding SS-OCT may look to characterize a partial PVD and correlate with clinical, ultrasound, and SDOCT findings, both pre and post vitrectomy.
Clinical examination at the slit-lamp is an efficient method and allows for the detection of a Weiss ring which indicates a complete PVD. However, in the absence of a Weiss ring, it may be difficult to ascertain the status of the vitreous overlying the fovea and the macula. Ultrasound allows for a kinetic examination and is the only method that can be used to assess the PVD status in an eye with a media opacity (ie, vitreous hemorrhage, dense cataract, corneal opacity). However, this method generally takes a longer time and can also be limited by the operator's abilities.

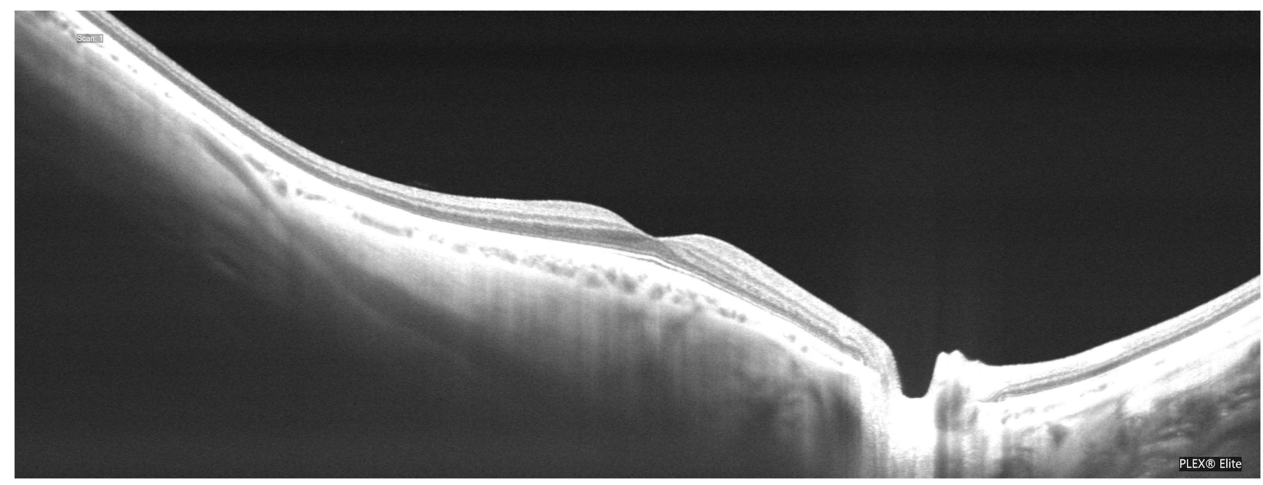

Figure 4 A complete PVD was identified by SS-OCT and ultrasound, but not by biomicroscopy. 


\section{Conclusions}

Because it produces a high level of detail of the vitreoretinal interface, SS-OCT, particularly with a wide window, can detect the presence or absence of complete PVD with high accuracy and reliability. Compared to biomicroscopy and ultrasound, SS-OCT may be a more sensitive modality for the detection of PVD in patients with clear media.

\section{Acknowledgments}

This manuscript was presented at Association for Research in Vision and Ophthalmology, Vancouver, BC, Canada on April 29, 2019.

\section{Funding}

NEI/NIH Core Grant P30EY019007, Research to Prevent Blindness Unrestricted Grant.

\section{Disclosure}

Royce W.S. Chen is a consultant for Allergan, Alimera and Carl Zeiss Meditec Inc. The other authors have no conflicts of interest to declare.

\section{References}

1. Lavinsky F, Lavinsky D. Novel perspectives on swept-source optical coherence tomography. Int J Retina Vitreous. 2016;2:25. doi:10.1186/ s40942-016-0050-y

2. Barteselli G, Bartsch D-U, Weinreb RN, et al. Real-time full-depth visualization of posterior ocular structures: comparison between full-depth imaging spectral domain optical coherence tomography and swept-source optical coherence tomography. Retina. 2016;36 (6):1153-1161. doi:10.1097/IAE.0000000000000842

3. Kishi S. Impact of swept source optical coherence tomography on ophthalmology. Taiwan J Ophthalmol. 2016;6(2):58-68. doi:10.1016/ j.tjo.2015.09.002

4. Itakura $\mathrm{H}$, Kishi $\mathrm{S}, \mathrm{Li} \mathrm{D}$, et al. Vitreous changes in high myopia observed by swept-source optical coherence tomography. Invest Ophthalmol Vis Sci. 2014;55(3):1447-1452. doi:10.1167/iovs.1313496

5. Bertelmann T, Goos C, Sekundo W, et al. Is optical coherence tomography a useful tool to objectively detect actual posterior vitreous adhesion status? Case Rep Ophthalmol Med. 2016;2016:3953147. doi:10.1155/2016/3953147

6. Johnson MW. Posterior vitreous detachment: evolution and role in macular disease. Retina. 2012;32(Suppl 2):S174-8. doi:10.1097/ IAE.0b013e31825bef62

Clinical Ophthalmology

\section{Publish your work in this journal}

Clinical Ophthalmology is an international, peer-reviewed journal covering all subspecialties within ophthalmology. Key topics include: Optometry; Visual science; Pharmacology and drug therapy in eye diseases; Basic Sciences; Primary and Secondary eye care; Patient Safety and Quality of Care Improvements. This journal is indexed on PubMed

Submit your manuscript here: https://www.dovepress.com/clinical-ophthalmology-journal
7. Johnson MW. Posterior vitreous detachment: evolution and complications of its early stages. Am J Ophthalmol. 2010;149(3):371-82 e1. doi:10.1016/j.ajo.2009.11.022

8. Uchino E, Uemura A, Ohba N. Initial stages of posterior vitreous detachment in healthy eyes of older persons evaluated by optical coherence tomography. Arch Ophthalmol. 2001;119(10):1475-1479. doi:10.1001/archopht.119.10.1475

9. Mojana F, Kozak I, Oster SF, et al. Observations by spectral-domain optical coherence tomography combined with simultaneous scanning laser ophthalmoscopy: imaging of the vitreous. Am J Ophthalmol. 2010;149(4):641-650. doi:10.1016/j.ajo.2009.11.016

10. Pang CE, Freund KB, Engelbert M. Enhanced vitreous imaging technique with spectral-domain optical coherence tomography for evaluation of posterior vitreous detachment. JAMA Ophthalmol. 2014;132(9):1148-1150. doi:10.1001/jamaophthalmol.2014.1037

11. Hwang ES, Kraker JA, Griffin KJ, et al. Accuracy of spectral-domain oct of the macula for detection of complete posterior vitreous detachment. Ophthalmol Retina. 2020;4(2):148-153. doi:10.1016/j. oret.2019.10.013

12. Moon SY, Park SP, Kim YK. Evaluation of posterior vitreous detachment using ultrasonography and optical coherence tomography. Acta Ophthalmol. 2020;98(1):p. e29-e35. doi:10.1111/aos.14189

13. Stanga PE, Sala-Puigdollers A, Caputo S, et al. In vivo imaging of cortical vitreous using 1050-nm swept-source deep range imaging optical coherence tomography. Am J Ophthalmol. 2014;157(2):397404 e2. doi:10.1016/j.ajo.2013.10.008

14. Schaal KB, Pang CE, Pozzoni MC, et al. The premacular bursa's shape revealed in vivo by swept-source optical coherence tomography. Ophthalmology. 2014;121(5):1020-1028. doi:10.1016/j. ophtha.2013.11.030

15. Itakura $\mathrm{H}$, et al. Observation of posterior precortical vitreous pocket using swept-source optical coherence tomography. Invest Ophthalmol Vis Sci. 2013;54(5):3102-3107. doi:10.1167/iovs.13-11769

16. Li D, Kishi S, Itakura H, et al. Posterior precortical vitreous pockets and connecting channels in children on swept-source optical coherence tomography. Invest Ophthalmol Vis Sci. 2014;55(4):2412-2416. doi:10.1167/iovs.14-13967

17. Tsukahara M, et al. Posterior vitreous detachment as observed by wide-angle OCT imaging. Ophthalmology. 2018;125(9):1372-1383. doi:10.1016/j.ophtha.2018.02.039

18. Kicova N, et al. Evaluation of a posterior vitreous detachment: a comparison of biomicroscopy, B-scan ultrasonography and optical coherence tomography to surgical findings with chromodissection. Acta Ophthalmol. 2012;90(4):e264-8. doi:10.1111/j.17553768.2011.02330.x

19. Chen KC, Jung JJ, Engelbert M. Swept source optical coherence tomography of the posterior vitreous after pars plana vitrectomy. Graefes Arch Clin Exp Ophthalmol. 2014;55(4):2412-2413. doi:10.1007/s00417-015-3018-y

20. Spaide RF. Visualization of the posterior vitreous with dynamic focusing and windowed averaging swept source optical coherence tomography. Am J Ophthalmol. 2014;158(6):1267-1274. doi:10.1016/j.ajo.2014.08.035
Central and CAS, and is the official journal of The Society of Clinical Ophthalmology (SCO). The manuscript management system is completely online and includes a very quick and fair peer-review system, which is all easy to use. Visit http://www.dovepress.com/ testimonials.php to read real quotes from published authors. 